\title{
Plague: a continuing threat
}

Background and epidemiology: An outbreak of plague in a remote diamond mine in the Democratic Republic of the Congo resulted in the deployment on Feb. 19, 2005, of a multidisciplinary team of epidemiologists, physicians and logisticians from Médecins Sans Frontières in collaboration with Medair, the World Health Organization and the Congolese Ministry of Health. By Mar. 30, when the team pulled out, they had confirmed 136 cases of pneumonic plague, a rare form of the disease caused by the bacterium Yersinia pestis; 57 were fatal.

Plague is a zoonosis of rodents that circulates mainly among small animals and their fleas. It is endemic in many countries in Africa, in the former Soviet Union, the Americas, including the southwestern United States, and parts of Asia (Fig. 1). There are 3 forms of infection: bubonic, septicemic and pneumonic. Bubonic plague is transmitted to humans through flea bites and direct contact with infected rodents. Domestic cats and dogs that have been in contact with rodents can transport the infected fleas. Septicemic plague occurs when bacteria enter the blood through flea bites or direct contact with infective material. Pneumonic plague, the most deadly form, occurs when bacteria infect the lungs either through septicemia or inhalation. Secondary pneumonia develops, and the bacteria can then be transmitted from person to person by respiratory droplets, which renders $Y$. pestis a potential bioterrorism agent.

In 2003, a total of 9 countries reported 2118 cases and 182 deaths. Nearly $99 \%$ of the cases and deaths were reported from Africa. The United States reports about $10-15$ sporadic cases a year, which typically result from single encounters with infected, wild rodents in rural settings. Outbreaks are rare and often indicate a breakdown in public health infrastructure and hygiene standards, such as that encountered in the context of poverty and the aftermath of warfare as experienced by the Congolese miners.

Clinical management: After an incubation period of 1-7 days, the patient experiences fever, chills, body aches, nausea and vomiting, marked prostration and delirium. ${ }^{1}$ The site of the flea bite is rarely detected, but regional lymphadenopathy is almost always present. The nodes, called "buboes," most often femoral but occasionally cervical or axillary, are swollen, painful and tender. The nodes are usually matted, and the surrounding tissue is edematous and frequently hemorrhagic. Infected nodes may drain to the skin. Primary septicemic plague may occur without lymphadenopathy. The incubation period for primary pneumonic plague is 2-3 days, and symptom onset is sudden and acute. If untreated, the case-mortality ratio is high.

The differential diagnosis for bubonic plague includes streptococcal and staphylococcal adenitis, tularemia and cat scratch disease. Pneumonic plague should be considered in the differential diagnosis of community-acquired pneumonia in areas where plague is endemic. Rapid diagnosis can be made by staining clinical specimens (e.g., bubo aspirates, blood, sputum) or by polymerase chain reaction, and it is confirmed by culture and serology. ${ }^{1}$ Presumptive treatment with streptomycin, tetracycline or doxycycline should be started without delay. Gentamicin may be substituted if streptomycin is not immediately available. $^{1}$
Prevention: Suspected clinical cases should be isolated and disinfected of fleas. If pneumonic plague is suspected, immediate contacts (face-to-face or household) should be disinfected and offered chemoprophylaxis with tetracycline or chloramphenicol for 1 week. Those refusing chemoprophylaxis should be quarantined and monitored for infection for 7 days.

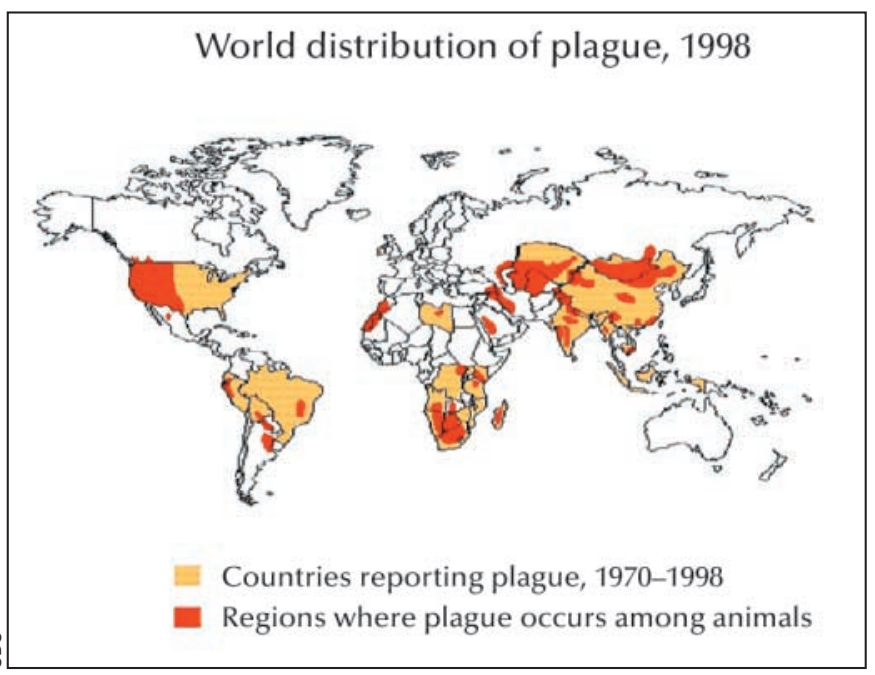

The mainstay of prevention is investment in a public health infrastructure to educate susceptible populations about the disease, maintain standards of hygiene, control rodent and flea populations and supply antibiotics when needed. A killed, whole-cell plague vaccine has been used in the past for select populations, but it offers poor protection against the pneumonic form of plague. Efforts are underway to develop a vaccine effective against both bubonic and pneumonic plague. ${ }^{2}$

\section{Erica Weir}

CMAF

\section{References \\ 1. Dennis D, Chow C. Plague. Pediatr Infect Dis f 2004;23:69-71. \\ 2. Titball R, Williamson ED. Vaccina- tion against bubonic and pneumonic plague. Vaccine 2001;19:4175-84.}

